Provided for non-commercial research and education use. Not for reproduction, distribution or commercial use.

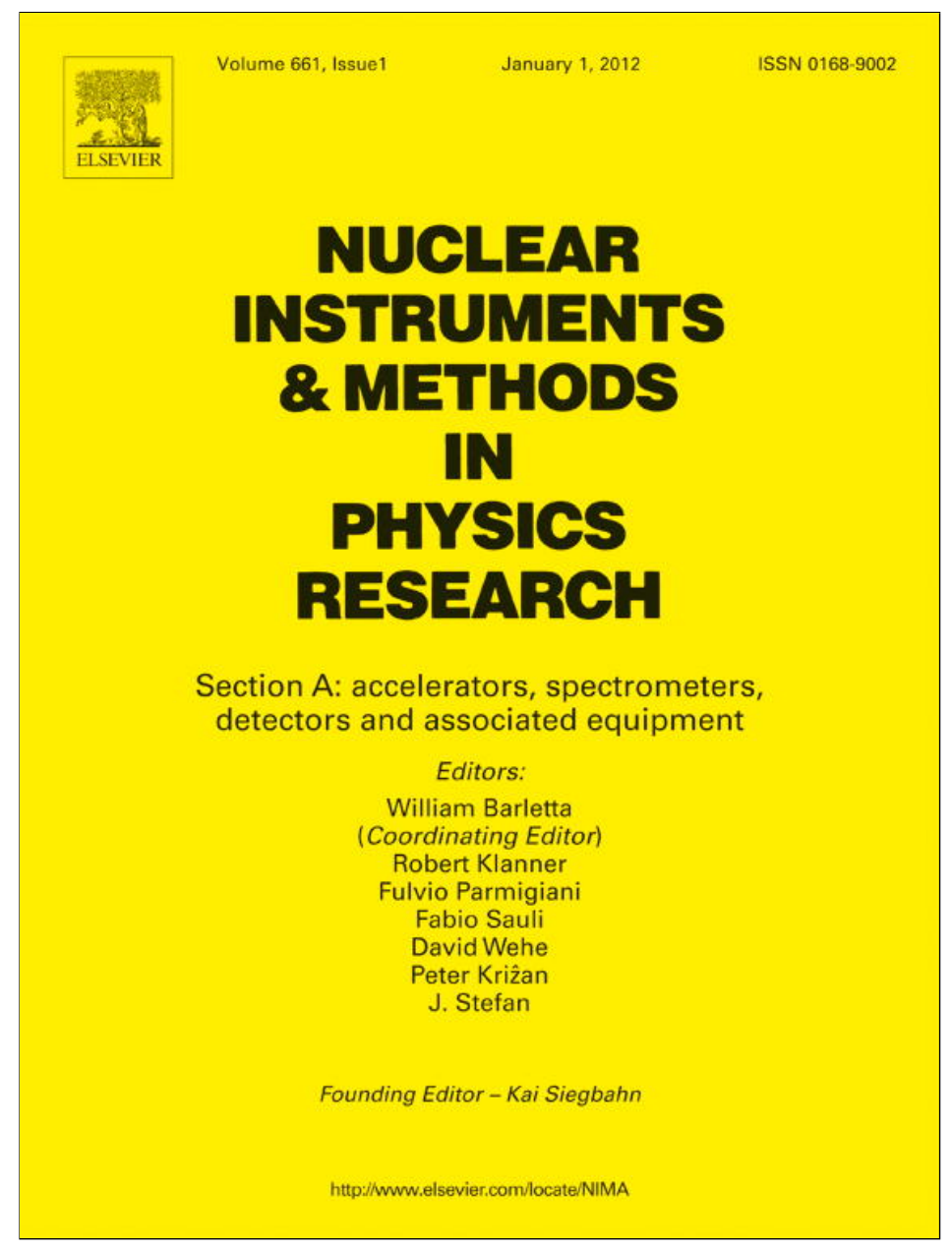

This article appeared in a journal published by Elsevier. The attached copy is furnished to the author for internal non-commercial research and education use, including for instruction at the authors institution and sharing with colleagues.

Other uses, including reproduction and distribution, or selling or licensing copies, or posting to personal, institutional or third party websites are prohibited.

In most cases authors are permitted to post their version of the article (e.g. in Word or Tex form) to their personal website or institutional repository. Authors requiring further information regarding Elsevier's archiving and manuscript policies are encouraged to visit:

http://www.elsevier.com/copyright 


\title{
Direct kinetic energy extraction from neutron Compton profiles
}

\author{
R. Senesi ${ }^{\mathrm{a}, \mathrm{b}, *}$ \\ a Università degli Studi di Roma “Tor Vergata”, Dipartimento di Fisica, Via della Ricerca Scientifica 1, 00133 Roma, Italy \\ ${ }^{\mathrm{b}}$ Centro NAST, Nanoscienze E' Nanotecnologie E' Strumentazione, Università degli Studi di Roma "Tor Vergata", Via della Ricerca Scientifica 1, 00133 Roma, Italy
}

\section{A R T I C L E I N F O}

\section{Article history:}

Received 18 August 2011

Received in revised form

16 September 2011

Accepted 20 September 2011

Available online 1 October 2011

Keywords:

Deep inelastic neutron scattering

Electronvolt neutron spectometers

Inelastic neutron scattering data analysis

Proton momentum distributions

\begin{abstract}
A B S T R A C T
Deep inelastic neutron scattering experiments provide access to atomic momentum distributions and mean kinetic energies. These quantities are intimately connected to nuclear quantum effects associated to the equilibrium ground state of condensed systems. The method to derive the single particle mean kinetic energy, directly employing the sum rules associated to the scattering functions, from a set of deep inelastic neutron scattering spectra is discussed. This method does not make use of nonlinear fitting of the scattering spectra.
\end{abstract}

(c) 2011 Elsevier B.V. All rights reserved.

\section{Introduction}

Inelastic neutron scattering experiments at high wave vector and energy transfers provide microscopic information on single particle microscopic dynamics of condensed matter systems [1]. In particular, when energy and wave vector transfers are in excess of about $1 \mathrm{eV}$ and $25 \AA^{-1}$, it is possible to reach the Deep Inelastic Neutron Scattering (DINS), or Neutron Compton Scattering (NCS), regime [2]. The latter represents a unique technique to probe the very short scale (time and distance) dynamics, measuring the single-particle momentum distribution $n(p)$ and mean kinetic energy, $\left\langle E_{K}\right\rangle$ of the scattering atoms [3,4]. In the DINS regime, the scattering is described within the framework of the Impulse Approximation (IA), which is exact in the limit of infinite momentum transfer [2,5]. This assumes that a single particle of the system, struck by the neutron, recoils freely from the collision, with inter-particle interaction in the final state being negligible (i.e. the wave function of the particle in its final state is a plane wave). Under these conditions, applying the momentum and energy conservation laws, the energy, $\hbar \omega$ and momentum, $\hbar \mathbf{q}$, transfers are related by

$\hbar \omega=\frac{\left(\mathbf{p}_{n}-\mathbf{p}_{n}^{\prime}\right)^{2}}{2 M}+\frac{\left(\mathbf{p}_{n}-\mathbf{p}_{n}^{\prime}\right) \cdot \mathbf{p}}{M}$

where $M$ and $\mathbf{p}$ are the mass and the momentum of struck particle before collision, while $\mathbf{p}_{n}$ and $\mathbf{p}_{n}^{\prime}$ are the momenta of the incident

*Correspondence address: Università degli Studi di Roma "Tor Vergata", Dipartimento di Fisica, Via della Ricerca Scientifica 1, 00133 Roma, Italy. Tel.: +3906 72594549; fax: +39062023507.

E-mail address: roberto.senesi@roma2.infn.it and scattered neutron (i.e. $\mathbf{p}_{n}-\mathbf{p}_{n}^{\prime}=\hbar \mathbf{q}$ ), respectively. Thus, the energy distribution of the scattered neutrons is directly related to the distribution of particle momenta parallel to the wave vector transfer. The IA can be regarded as a special case of the incoherent approximation, where, in the case of high energy collisions, a short time $(t \longrightarrow 0)$ expansion of the atomic position operator, $\mathbf{R}(t)$ is applied to the position operator of the struck atom of mass $M$ and momentum $\mathbf{p}: \mathbf{R}(t)=\mathbf{R}(0)+(t / M) \mathbf{p}$ [5]. Within the framework of the IA, the dynamical structure factor is described by

$S_{I A}(\mathbf{q}, \omega)=\hbar \int n(\mathbf{p}) \delta\left[\hbar \omega-\hbar \omega_{r}-\frac{\mathbf{p} \cdot \hbar \mathbf{q}}{M}\right] d \mathbf{p}$

where $n(\mathbf{p})$ is the atomic momentum distribution, and $\hbar \omega_{r}$ is the recoil energy:

$\hbar \omega_{r}=\frac{\hbar^{2} q^{2}}{2 M}$

The physical implication of Eq. (1.2) is that scattering occurs between the neutron and a single particle, with conservation of kinetic energy and momentum of the particle+neutron system, as introduced in Eq. (1.1). If the IA is valid, the two dynamic variables $\omega$ and $q$ can be explicitly coupled through the definition of the West scaling variable $y$ as [5]

$y=\frac{M}{\hbar^{2} q}\left(\hbar \omega-\hbar \omega_{r}\right)$.

Eq. (1.2) can be then reduced to the form

$S_{I A}(\mathbf{q}, \omega)=\frac{M}{\hbar q} J(y, \hat{q})$ 
where

$$
J(y, \hat{q})=\hbar \int n\left(\mathbf{p}^{\prime}\right) \delta\left(\hbar y-\mathbf{p}^{\prime} \cdot \hat{q}\right) d \mathbf{p}^{\prime} .
$$

$J(y, \hat{q})$ is the Neutron Compton Profile. The quantity $\hat{q}$ is a unit vector, as $J(y, \hat{q})$ no longer depends on the magnitude of q. The function $J(y, \hat{q}) d y$ represents the probability for an atom to have a momentum parallel to $\hat{q}$ of a magnitude between $\hbar y$ and $\hbar(y+d y)$. In an isotropic system, the direction $\hat{q}$ is immaterial and Eq. (1.6) becomes

$J(y)=2 \pi \hbar \int_{|\hbar y|}^{\infty} p n(p) d p$.

The single particle mean kinetic energy is $\left\langle E_{K}\right\rangle=\left(3 \hbar^{2} / 2 M\right) \int_{-\infty}^{\infty} y^{2}$ $J_{I A}(y) d y=\left(3 \hbar^{2} / 2 M\right) \sigma^{2}$. The deviations from the IA which occur at finite $q$ are generally referred to as Final State Effects (FSE). FSE are due to the localization of the struck atom in its final state, by surrounding atoms. These make the momentum in the final state uncertain and since the dynamic structure factor, in Eq. (1.2), is determined by Fourier transform of the product of initial and final state wave functions, the momentum in the initial state is also uncertain. The overall effect is a broadening of the observed $J(y)$, which is similar to that introduced by an instrumental resolution effect. When FSE are present, the Neutron Compton Profile becomes $q$ dependent i.e. $J(y)$ needs to be replaced by $J(y, q)$, so that Eq. (1.5) is replaced by

$J(y, q)=\frac{\hbar q}{M} S(q, \omega)$.

Representing the FSE as a series in powers of $1 / q$, one finds for the isotropic case according to Sears [6]

$J(y, q)=J(y)-\frac{A_{3}}{q} \frac{d^{3}}{d y^{3}} J(y)+\frac{A_{4}}{q^{2}} \frac{d^{4}}{d y^{4}} J(y)+\cdots$

where the coefficients $A_{n}$ are related to the interatomic potential [6]. It has to be stressed that the sum rules obeyed by $J(y, q)$ are directly related to the sum rules of the incoherent dynamical structure factor, which are preserved under the West-scaling transformation of Eq. (1.5) [6]. Systematic studies of the zero-point atomic kinetic energies allow an insight into the relevance of quantum effects in the energetics of monoatomic and molecular systems. At the same time, accurate studies of momentum distribution line shapes provide unique information on the local environment and potential energy surfaces experienced by the target atom. Indeed the behavior of protons, and more generally of light nuclei in condensed phases, is significantly affected by quantum effects even at ambient temperatures. The isotopic effect in water, the ferroelectric behavior of potassium diphosphate, and the formation of high pressure ice phases, are just a few of the relevant phenomena where the quantum behavior of the nuclei plays a role. Because of the noncommuting character of position and momentum operators in quantum mechanics, $n(p)$ and $\left\langle E_{K}\right\rangle$ are very sensitive to the local environment. A typical example is the dependence of $\left\langle E_{K}\right\rangle$ on the macroscopic density in monoatomic liquids and solids, such as ${ }^{4} \mathrm{He}$ [7-9] or ${ }^{3} \mathrm{He}$ [10]. This is a genuine quantum effect, where the increased space localization of the atoms in the high density liquid or solid phases, with reduced fluctuations in position space, results in increased fluctuations in momentum space, with an excess of kinetic energy due to localization, following the Heisenberg indetermination principle. At the same time molecular systems, including water, in nanoscale confinement, show a variety of responses in terms of $n(p)$ and $\left\langle E_{K}\right\rangle$, due to the localization and/or delocalization effects induced by the interaction with the confining substrate [11-13]. Moreover, recent theoretical studies in bulk water have been focussed in the quantum interplay between position and momentum spaces responses in the phases of ice [14]. Therefore, systematic studies of the proton mean kinetic energy in bulk liquid water from the supercooled metastable phase to supercritical conditions highlight the connection between the proton short-scale response to the changes in the hydrogen bond network [15-25]. At the same time, studies of the proton kinetic energies of water in a variety of settings have provided an insight into the response of the quantum state of water's protons to the perturbation induced by nanoscale confinement or a macromolecular surface [26-29]. Neutron scattering experiments in the DINS regime are routinely carried out on the Vesuvio time of flight electronvolt spectrometer at the ISIS pulsed neutron source [30,31]. Here the scattering occurs in the range $2.5 \mathrm{eV} \leq \hbar \omega_{r} \leq 30 \mathrm{eV}$. The instrument is equipped with 64 scintillators in the forward scattering angles $\left(\vartheta \leq 75^{\circ}\right)$ and 132 scintillators in backscattering range $\left(130^{\circ} \leq \vartheta \leq 165^{\circ}\right)$. At each scattering angle the energy of the scattered neutrons, $E_{1}$, is selected by using $\mathrm{Au}$ analyzers $\left(E_{1}=4897 \mathrm{meV}\right)$. The time of flight spectrum is obtained by differencing techniques that allow the removal of the sample-independent background and a sharpening of the resolution with respect to a single measurement [32-41]. Data reduction on time of flight spectra is then carried out using procedures allowing to correct for multiple scattering [42], and heavy atom signals, such as oxygen and aluminum from the sample containers, in order to isolate the single scattering from the light element of interest, e.g. the proton, deuteron, ${ }^{3} \mathrm{He}$, etc. [31]. After the above corrections a time of flight spectrum contains the single scattering contribution from the isolated mass of interest and is therefore related to the neutron Compton profile by

$F_{l}(y, q)=\frac{B M}{E_{0} \Phi\left(E_{0}\right)} q C_{l}(t, \vartheta)$

where $F_{l}(y, q)$ is the fixed-angle experimental Compton profile for the $l$-th detector, $E_{0}$ is the incident neutron energy, $\Phi\left(E_{0}\right)$ is the energy-dependent incident neutron fluence rate, $C_{l}(t, \vartheta)$ is the countrate at the $l$-th detector, and $B$ is a constant taking into account the detection solid angle, the detector efficiency at $E=E_{1}$, the timeenergy Jacobian, the free-atom neutron-proton cross-section, the number of protons hit by the neutron beam [2]. The expression above implies the following representation of $F_{l}(y, q)$, namely [2]:

$F_{l}(y, q)=\left[J(y)+\Delta J_{l}(y, q)\right] \otimes R_{l}(y, q)$.

$\Delta J_{l}(y, q)$ are the $q$-dependent deviations from the impulse approximation (Final State Effects, FSE), and $R_{l}(y, q)$ is the fixed-angle instrumental resolution function. The latter expression is commonly adopted and assessed to represent DINS spectra using both directgeometry chopper spectrometers [9,43-48], as well as on inverse geometry instruments such as VESUVIO $[2,21,49]$. In some cases the additive form for representing Final State Effects has been compared with a convolution form that used a broadening function to account for FSE, indeed providing the same results within the experimental uncertainty $[45,50]$. Moreover, a number of works have assessed the validity of representing the instrumental broadening effects by the convolution with a resolution function such as $R_{l}(y, q)$ [32,51,52]. The DINS data analysis is generally carried out using parametric methods, such as nonlinear fitting of the $F_{l}(y, q)$, using model functions for $J(y)$. The most relevant model functions used are the Gauss-Hermite expansion [2]

$J(y)=\frac{e^{-y^{2} / 2 \sigma^{2}}}{\sqrt{2 \pi} \sigma}\left[1+\sum_{n=2}^{\infty} \frac{a_{n}}{2^{2 n} n !} H_{2 n}\left(\frac{y}{\sqrt{2} \sigma}\right)\right]$

where $\sigma$ is the standard deviation of $J(y), H_{2 n}$ are the Hermite polynomials and $a_{n}$ is the corresponding coefficients or the spherically averaged multivariate Gaussian for molecular systems [25], where the following expression is assumed:

$n(p)=\left\langle\frac{1}{\sqrt{8 \pi^{3}} \sigma_{z} \sigma_{x} \sigma_{y}} \exp \left(-\frac{p_{z}^{2}}{2 \sigma_{z}^{2}}-\frac{p_{x}^{2}}{2 \sigma_{x}^{2}}-\frac{p_{y}^{2}}{2 \sigma_{y}^{2}}\right)\right\rangle_{\Omega}$ 
where $\sigma_{i}$ are the standard deviations along the principal axes of the molecular frame of reference. In all the cases above, the fitting parameters are the set of $\sigma, a_{n}, \sigma_{i}$, and the FSE coefficients $A_{n}$. The fit relies on the minimization of

$\chi^{2}=\sum_{l} \sum_{i} \frac{\left(F_{l}^{\text {th }}\left(y_{i}, q_{i}\right)-F_{l}\left(y_{i}, q_{i}\right)\right)^{2}}{\varepsilon_{l, i}^{2}}$

where the model function is $F_{l}^{\text {th }}\left(y_{i}, q_{i}\right)=\left[J\left(y_{i}\right)+\Delta J_{l}\left(y_{i}, q_{i}\right)\right] \otimes R_{l}\left(y_{i}, q_{i}\right)$. Here the index $l$ represents the detector index, the index $i$ represents the $y$ value at the $i$-th bin, and $\varepsilon_{l i}^{2}$ is the error for each data point.

The aim of this work is to discuss and provide the basis of a different, non-parametric method, to determine $\sigma$, i.e. from the numerical calculation of the variance of experimental Compton profiles, $F_{l}(y, q)$, and of the detector-averaged Compton profile, $\bar{F}(y)=\sum_{l=1}^{n} F_{l}(y, q) 1 / n$, where $n$ is the total number of detectors. This method has been successfully applied in previous works to bulk helium [48] and, more recently, to confined molecular hydrogen [12], and ice [53]. In particular, the latter work made use of a recent theoretical study on proton momentum distribution [54], where a quantity named mean force was proposed to access the details of three-dimensional momentum distributions; the mean force can be directly calculated from DINS data to provide a further independent and non-parametric estimate of $\sigma$. However, the above works did not discuss in details the basis of applicability of the method by providing proofs of concept and demonstration, in terms of sum rules and properties of the scattering function. The work presented here aims at providing the above basis for an assessment of the method in views of its routine use in experiments. The advantage of this method is a direct access to the single particle mean kinetic energy without resorting to parametric, nonlinear fitting. The variance obtained in this way can be used as a model-free constraint in the fitting, providing physical limits to $\left\langle E_{K}\right\rangle$, or be used as a final determination of $\left\langle E_{K}\right\rangle$ in high statistical quality experimental data. Indeed, the use of $\bar{F}(y)$ is particularly useful on Vesuvio, since averaging the spectra allows one to reduce the error bar on individual data points, but more importantly helps in reducing the effects of noise. Ref. [36] accurately shows that the count rate at a particular detector is obtained from a differencing technique between two large count rates, one with the energy analyzer between sample and detector, and one without it. Data points are thus affected by counting errors which are of similar magnitudes at the peak and on the tails of the spectrum, as well as by noise, which is more pronounced at high positive $y$ values. The evaluation of $\bar{F}(y)$ allows to average the noise and to reduce it close to its mean (zero) value. This property can be exploited to calculate the second moment, which is very sensitive to the tails of the experimental spectra. In Section 2 the sum rules and the convolution approach behind the method are presented, while in Section 3 the method is applied to Monte Carlo simulation of $F_{l}(y, q)$ and $\bar{F}(y)$ spectra, to provide a proof of concept of the method. Section 4 reports the discussion and conclusions.

\section{Sum rules and convolution approach}

The sum rules for the neutron Compton profile derive from the general sum rules of the incoherent dynamical structure factor, with special regards to its Impulse Approximation limit, $S_{I A}(\mathbf{q}, \omega)$ [6]. The most relevant are

$\int_{-\infty}^{\infty} d y J(y)=1$

$\int_{-\infty}^{\infty} d y y J(y)=0=J_{1}(y)$ $\int_{-\infty}^{\infty} d y J(y) y^{2}=\sigma^{2}$

While the sum rules for the final state effects are [6]

$\int_{-\infty}^{\infty} d y \Delta J(y, q)=0=\Delta J_{0}(y, q)$

$\int_{-\infty}^{\infty} d y y \Delta J(y, q)=0=\Delta J_{1}(y, q)$

$\int_{-\infty}^{\infty} d y \Delta J(y, q) y^{2}=0=\Delta J_{2}(y, q)$.

Therefore, for finite wave vector transfers, the sum rules valid for the incoherent dynamical structure factors retain their validity $[5,55]$, and the following sum rules apply for $J(y, q)$ :

$\int_{-\infty}^{\infty} d y J(y, q)=1$

$\int_{-\infty}^{\infty} d y y J(y, q)=0$

$\int_{-\infty}^{\infty} d y J(y, q) y^{2}=\sigma^{2}$

While $J(y)$ is a probability density distribution (longitudinal momentum distribution), with unitary zeroth moment, zero first moment, and second moment equal to $\sigma^{2}$, for any line shape of the underlying momentum distribution, the FSE corrections must have zeroth, first, and second moments equal to zero to satisfy the sum rules of the incoherent dynamical structure factor [56]. The resolution function depends on the spectrometer, in particular on the q, $\omega$ values accessed by a particular detector, with index $l$, and their relative uncertainties. In any circumstances the following sum rules apply:

$\int_{-\infty}^{\infty} d y R_{l}(y, q)=1$

$\int_{-\infty}^{\infty} d y R_{l}(y, q) y^{2}=\sigma_{l}^{2}$

It has to be stressed that, thanks to the improvements in detector and energy analysis concepts, namely the Foil Cycling Technique applied to resonance detectors $[34,35,38,41]$, the resolution function has a finite second moment, at variance with the previous setup using the Single Difference method [2] that provided a resolution function of Voigt line shape with Lorentzian tails with virtually infinite second moment. In the kinematical range $q, \omega$ accessed by fixed detector spectra, it as been shown that, for the $q$-dependent quantities $J(y, q)$ and $R_{l}(y, q)$, the zeroth-order sum rules are valid within $5 \times 10^{-5}$ [57], while the correspondent accuracy for secondorder sum rules are presented in this work.

The same sum rules are now examined in the context of the convolution representation of the experimental Compton profile $F_{l}(y, q)$ in Eq. (1.11). We first recall the moments of the convolution of two real functions, $f_{1}(x)$, and $f_{2}(x)$, having finite zeroth, first and second moments [58]. Following the notations of Ref. [58], we have

$h(x)=f_{1}(x) \otimes f_{2}(x)$.

The zeroth moment of $h(x)$ is

$Z_{h}=Z_{1} \cdot Z_{2}$

where $Z_{1}$ and $Z_{2}$ are the zeroth moments of $f_{1}(x)$ and $f_{2}(x)$, respectively. The first moment of $h(x)$ is

$F_{h}=F_{1} \cdot Z_{2}+Z_{1} \cdot F_{2}$ 
where $F_{1}$ and $F_{2}$ are the first moments of $f_{1}(x)$ and $f_{2}(x)$, respectively. The second moment of $h(x)$ is

$S_{h}=S_{1} \cdot Z_{2}+2 F_{1} \cdot F_{2}+Z_{1} \cdot S_{2}$

where $S_{1}$ and $S_{2}$ are the second moments of $f_{1}(x)$ and $f_{2}(x)$, respectively. In applying the above formulas to the case of the two functions $J(y, q)$ and $R_{l}(y, q)$, we can also make use of the distributive property of the convolution, since $J(y, q)=J(y)+\Delta J(y, q)$. Therefore, the zeroth and second moments of $F_{l}(y, q)$ are

$\int_{-\infty}^{\infty} d y F_{l}(y, q)=1$

$\int_{-\infty}^{\infty} d y F_{l}(y, q) y^{2}=\sigma^{2}+\sigma_{l}^{2}$.

These results are valid for any momentum distribution line shape as they derive from the definition of probability density distributions with finite variance. The same formalism can be thus applied to the angle-averaged or detector-averaged experimental neutron Compton profile:

$\bar{F}(y)=\sum_{l=1}^{n} F_{l}(y, q) \frac{1}{n}$

where $n$ is the total number of detectors. In the above average, the $q$ dependence is smeared as the contributions from different detectors accessing different $q$ values for the same $y$ value are averaged [57]. Using the same arguments as before it can be demonstrated that

$\int_{-\infty}^{\infty} d y \bar{F}(y)=1$

$\int_{-\infty}^{\infty} d y \bar{F}(y) y^{2}=\sigma^{2}+\left\langle\sigma_{l}^{2}\right\rangle$.

Thus the quantity $\bar{F}(y)$ conveys information on the variance of the momentum distribution and on the single particle kinetic energy, similar to the variances obtained from individual detectors.

\section{Monte Carlo simulation of $F_{l}(y, q)$ and $\bar{F}(y)$ spectra and determination of mean kinetic energy}

The method of direct integration of $y^{2} \bar{F}(y)$ has been applied to a set of Monte Carlo simulated experimental Compton profiles for a proton-containing system. The DINSMS code [42] is routinely used for data reduction on Vesuvio, in particular for the evaluation of multiple scattering contributions and for the determination of the resolution. The code incorporates the resolution in a virtually exact manner, without relying on the convolution approximation, tracking and weighting individual neutron histories by probability distribution function for the geometrical uncertainties, emission time from the moderator, energy analyzer transfer function, etc. Applying the method to such synthetic data is a self-consistent test providing a proof of concept. The simulations presented here were carried out with a modification to the DINSMS code that takes into account the Foil Cycling transfer function and the time and efficiency responses of the YAP detectors, as currently employed on Vesuvio. The sample was monoatomic hydrogen with isotropic gaussian momentum distribution with standard deviation $\sigma_{M C}=4.7 \AA^{-1}$, corresponding to $\left\langle E_{K}\right\rangle=138 \mathrm{meV}$.

Final state effects were also simulated, assuming an isotropic harmonic proton effective potential with mean frequency $\hbar \bar{\omega}=275 \mathrm{meV}$, including terms up to $1 / q^{2}$ in the FSE expansion. The quantity chosen are typical of protons in liquid water around room temperature. Data have been simulated for $10^{6}$ neutron histories for each detector, for a subset, 32 instead of 64 , of the
Vesuvio forward detector bank, without loss of generality. The resolution has been determined by simulating a hydrogen containing sample with isotropic Gaussian momentum distribution with $\sigma_{R}=5.0 \times 10^{-3} \AA^{-1}$ and simulating $10^{9}$ events for each detector. An example of time of flight simulated data for a subset of detectors is reported in Fig. 1. Data have been then transformed in longitudinal proton momentum, $y$, to provide the fixed angle experimental Compton profiles, $F_{l}(y, q)$ as reported in Fig. 2. A selfconsistent test of the convolution approximation was first carried out by fitting the set of $F_{l}(y, q)$ spectra by minimizing the chisquared in Eq. (1.14), using the model function

$F_{l}^{t h}\left(y_{i}, q_{i}\right)=\left[\frac{e^{-y_{i}^{2} / 2 \sigma^{2}}}{\sqrt{2 \pi} \sigma}\left[1+\frac{c_{1}}{q_{i}} \cdot H_{3}\left(\frac{y_{i}}{\sqrt{2} \sigma}\right)\right]\right] \otimes R_{l}\left(y_{i}, q_{i}\right)$

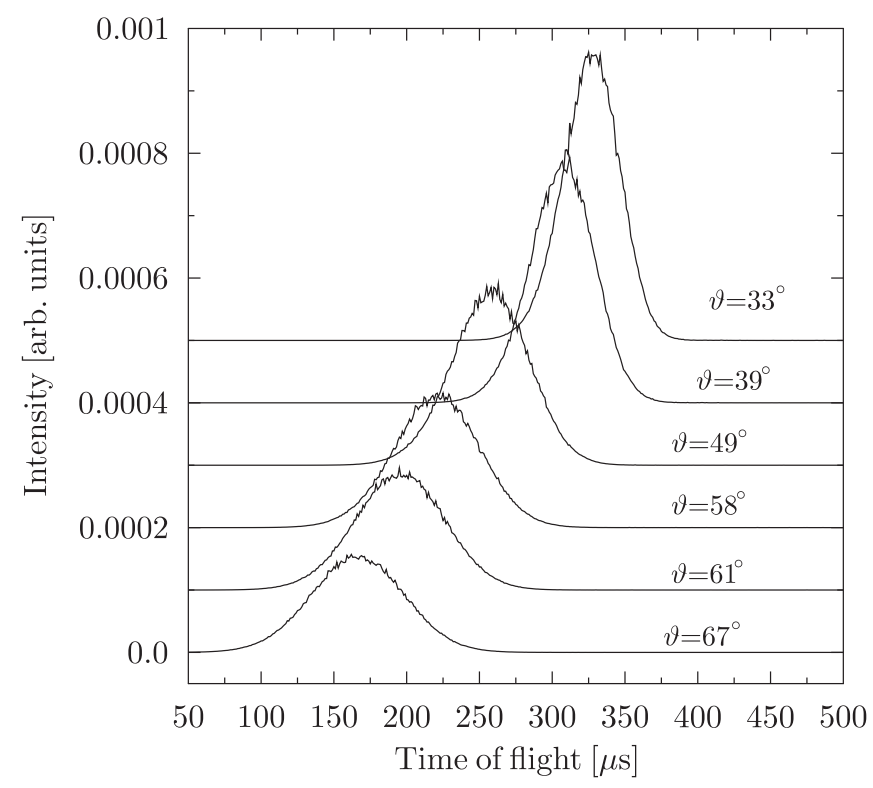

Fig. 1. Time of flight spectra of Monte Carlo simulated data for a subset of detectors (counting errors omitted). With the exception of the detector at $\vartheta=67^{\circ}$, all spectra are shifted upwards for clarity.

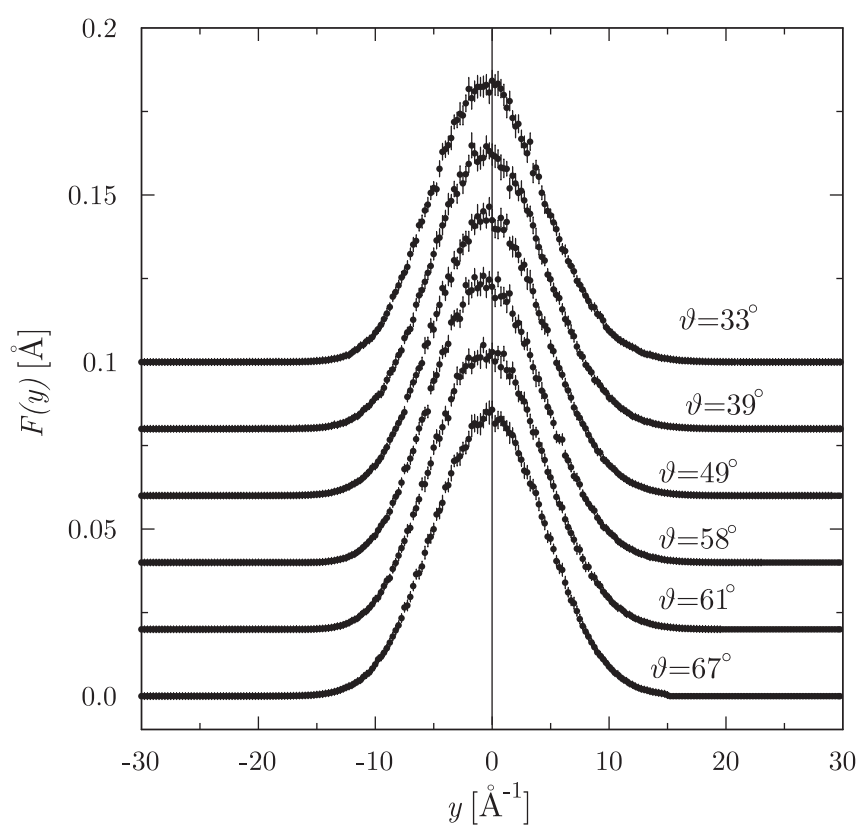

Fig. 2. $F_{l}(y, q)$ spectra of Monte Carlo simulated data for a subset of detectors. With the exception of the detector at $\vartheta=67^{\circ}$, all spectra are shifted upwards for clarity. 
where $\sigma$ and $c_{1}$ are fitting parameters; the $c_{1}$ parameter represents the Final State Effects coefficient, in the following representation:

$\Delta J(y, q)=\frac{e^{-y^{2} / 2 \sigma^{2}}}{\sqrt{2 \pi} \sigma} \frac{c_{1}}{q} H_{3}\left(\frac{y}{\sqrt{2} \sigma}\right)$

where

$c_{1}=\frac{M^{2} \bar{\omega}^{2}}{12 \hbar^{2}} \frac{1}{\sigma^{3} 2^{3 / 2}}$

and $M$ is the proton mass [6]. The fit yielded $\sigma=(4.71 \pm 0.04) \AA^{-1}$, $c_{1}=(0.5 \pm 0.1) \AA$, with a corresponding $\left\langle E_{K}\right\rangle=(139 \pm 2) \mathrm{meV}$, in agreement with the input value of the Monte Carlo simulations. The $c_{1}$ value in the fit is also in good agreement with the input value used in the simulation, that, based on the harmonic frequency $\hbar \bar{\omega}=275 \mathrm{meV}$, gives, for the coefficient of the $1 / q$ term, $c_{1}=0.62 \AA$. Besides providing a self-consistent assessment of the convolution approximation of Eq. (1.11), this result provides a stringent test of the methods for estimating the resolution from Monte Carlo simulations. The uncertainty of $+2 \mathrm{meV}$ can be regarded as the typical value of uncertainty from parametric estimations of the proton mean kinetic energy from high quality DINS data on the Vesuvio spectrometer.

The aim of the method outlined in this work is to apply the direct integration of the neutron Compton profile to the detectoraveraged signal. The detector-averaged spectrum, $\bar{F}(y)$, and the corresponding detector-averaged resolution, $\bar{R}(y)$ are reported in Fig. 3.

From this figure it can be noticed that a residual asymmetry is present in $\bar{F}(y)$, but this is not surprising since Final State effects are not canceled out by the average over scattering angles (i.e. detectors). The ratio between the FWHM of $\bar{F}(y)$ and $\bar{R}(y)$ is approximately $17 \%$.

In Fig. 4 the "kinetic energy distribution", $y^{2} \bar{F}(y)$, is reported, together with the corresponding quantity for the resolution, $y^{2} \bar{R}(y)$.

This figure allows one to appreciate the relative importance of the underlying momentum distribution and that of the spectrometer's resolution in the evaluation of the proton mean kinetic

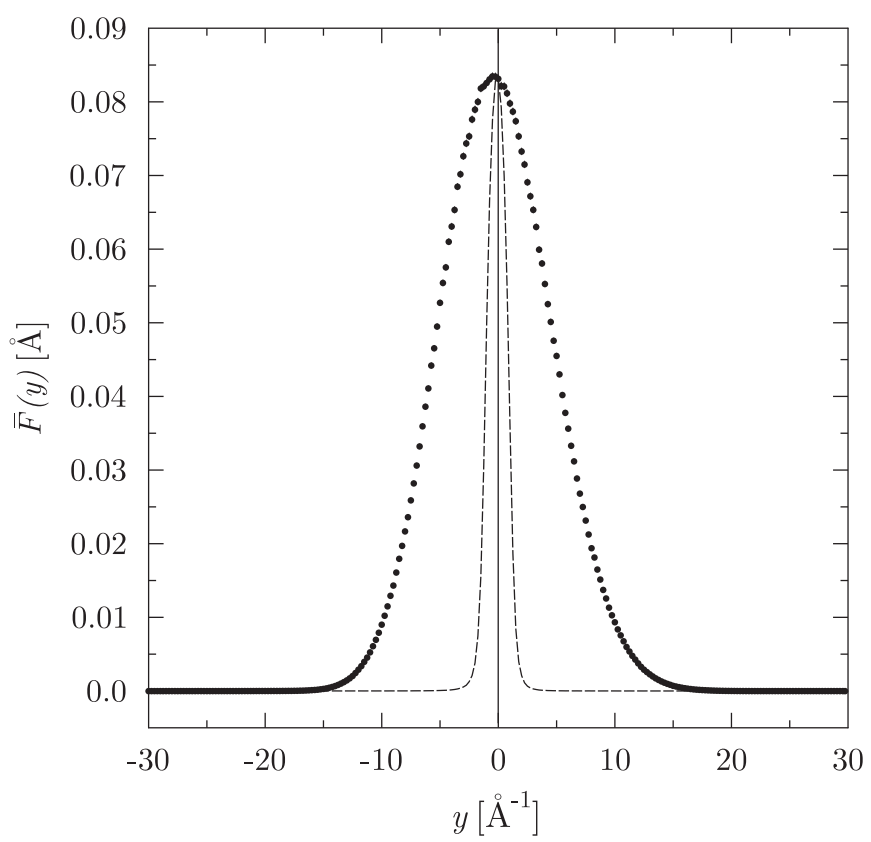

Fig. 3. Detector-averaged spectrum, $\bar{F}(y)$ (dots with error bars), and detectoraveraged resolution, $\bar{R}(y)$ (dashed line). The latter is normalized to the peak height of $\bar{F}(y)$.

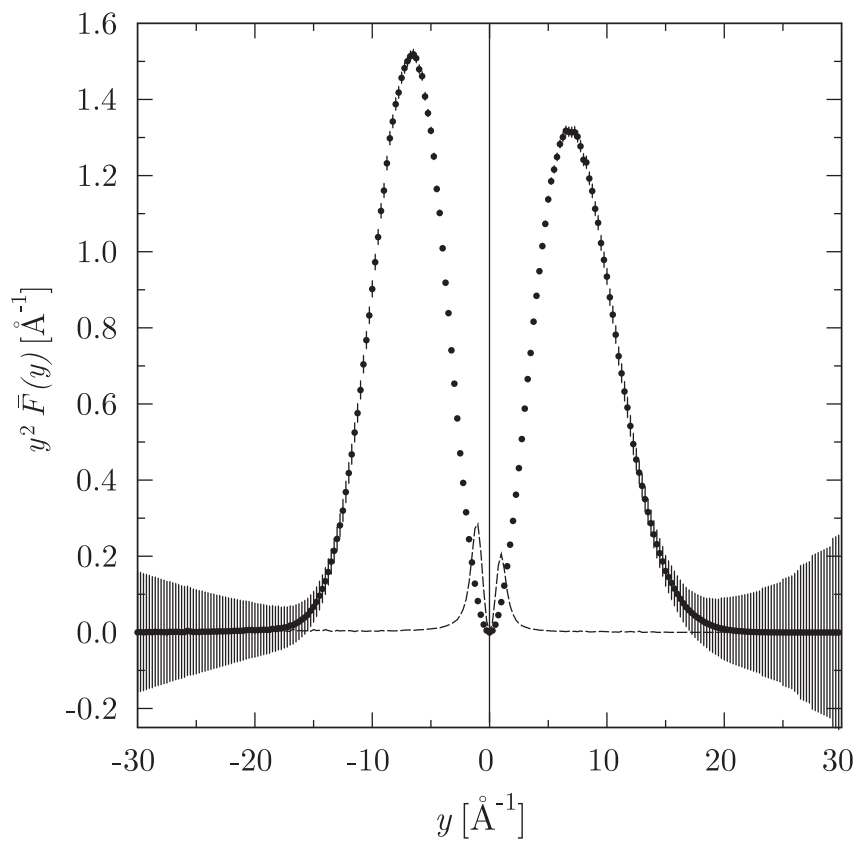

Fig. 4. Kinetic energy distribution, $y^{2} \bar{F}(y)$ (dots with error bars), and the same quantity evaluated for the detector-averaged resolution, $y^{2} \bar{R}(y)$ (dashed line). At variance with Fig. 3, $\bar{R}(y)$ is normalized to unit area, following the sum rules.

energy: for DINS scattering from protons, the former is the main contribution to the variance of the experimental Compton profile, while the variance of the resolution is a small percentage. It can be noticed that the left-right asymmetry in $y^{2} \bar{F}(y)$ results from both the presence of final state effects, and form a small asymmetry in the resolution line shape. This is the main contribution that accounts for any deviation of Eq. (2.11) from being exact. Numerical integration of $y^{2} \bar{F}(y)$ and of $y^{2} \bar{R}(y)$ resulted in: $y^{2} \bar{F}(y)=(22.6 \pm 0.32) \AA^{-2}$, and $y^{2} \bar{R}(y)=0.78 \AA^{-2}$, thus confirming a $\simeq 3 \%$ contribution of the resolution to the variance of the experimental signal. The results for the standard deviation and mean kinetic energy are: $\sigma=(4.67 \pm$ $0.03) \AA^{-1}$ and $\left\langle E_{K}\right\rangle=(137 \pm 2) \mathrm{meV}$. These quantities are in agreement, within uncertainty, to the input values of the Monte Carlo simulation.

It is of particular importance, from the experimental point of view, that the role played by the experimental noise in real data may modify the value as well as the uncertainty on the derivation of $\left\langle E_{K}\right\rangle$. For example, in Fig. 4 it can be noticed that for $|y| \geq$ $20 \AA^{-1}$, the tails of the kinetic energy distribution are zero within error and do not contribute to $\left\langle E_{K}\right\rangle$. Therefore, from a conservative point of view, an integration in the range $-4.25 \sigma \leq y \leq 4.25 \sigma$ would be sufficient to evaluate $\left\langle E_{K}\right\rangle$. In other words, if the FWHM of $\bar{F}(y)$ is a factor $\geq 6$ larger than the FWHM of $\bar{R}(y)$ (6.5 in this case), then an integration range within $|y| \geq 20 \AA^{-1}$ can be safely chosen.

Based on previous experience [53], on VESUVIO the noise for $|y| \geq 25 \AA^{-1}$ induces fluctuations on the values of $y^{2} \bar{F}(y)$ of the order of $\pm 0.5 \AA^{-2}$. Moreover, the noise has a left-right asymmetry due to the fact that large positive $y$ correspond to bin widths with large incident energy width, due to the energy-time of flight Jacobian. Therefore, these are more affected by fluctuations compared to negative $y$ values. While a realistic simulation of this type of noise, as well as the simulation of gamma/neutron backgrounds in time of flight spectra, is beyond the capability of the present simulation code, it is possible to provide estimates 
of the quality of the experimental $\bar{F}(y)$ for reliable estimates of $\left\langle E_{K}\right\rangle$ that provide an uncertainty of the order of $\leq 10 \%$ on the kinetic energy: (1) the counting statistics has to be characterized by approximately $1 \%$ error at the centroid of $\bar{F}(y)$ and of approximately $15 \%$ at $1 / 15$ of the peak height, respectively; (2) given the current resolution for protons, if the FWHM of $\bar{F}(y)$ is a factor $\geq 6$ larger than the FWHM of $\bar{R}(y)$, then the integration of $y^{2} \bar{F}(y)$ in the range $-20 \AA^{-1} \leq y \leq 20 \AA^{-1}$ would provide a reliable estimate of $\left\langle E_{K}\right\rangle$.

These results show that the non-parametric determination of the mean kinetic energy, through direct integration applied together with the convolution approximation of the experimental resolution, can be used to derive $\left\langle E_{K}\right\rangle$ of the proton, as well as heavier elements, in condensed matter systems. It appears that the sum rules applied to the detector-averaged neutron Compton profile introduce a systematic underestimations of the order of $0.6 \%$ on the standard deviation, and $0.7 \%$ on the proton kinetic energy, well within the typical experimental uncertainties. This calculation can offer an additional data analysis tool for deep inelastic neutron scattering experiments that, without resorting to any bias induced by parametric data analysis, allows one to derive the quantum zero-point kinetic energies.

\section{Discussion and conclusions}

Deep inelastic neutron scattering studies have undergone remarkable improvements in the last five years, thanks to the efforts in research and development on energy analysis and devices, together with novel theoretical approaches allowing a direct and quantitative comparison between theory and experiment. These efforts have been focussed mainly on momentum distribution and mean kinetic energy of the proton in hydrogenbonded systems, where the role of anharmonic inter-particle effective potentials and anisotropic harmonic potentials can be investigated in great details. The interest in non-parametric methods of analysis of DINS data relies on the possibility of accessing details of the proton local environment with recently proposed quantities, such as the proton mean force [54], that are derived from the experimental neutron Compton profiles, and are sensitive to the anisotropic-harmonic or anharmonic character of the proton motion along hydrogen bonds. The method described here can be applied to isotropically averaged samples such as liquids or polycrystalline materials, but can be further extended to oriented and monocrystalline samples. The determination of $\left\langle E_{K}\right\rangle$ from the direct integration of the neutron Compton profile introduces a systematic underestimate that is well below the typical uncertainties of experiments. The proof of concept provided by the analysis of Monte Carlo simulated data demonstrates that the mean kinetic energy can be reliably determined without resorting to parametric fitting methods. The method applied to simulated data does not however take into account the role of noise, which is inevitably present in real experiment. It appears therefore that a necessary prerequisite for the application of the method presented here is the recording of experimental data sets with high statistical quality, such as those currently possible on Vesuvio, or with any future $\mathrm{eV}$ neutron spectrometer at next generation spallation sources.

\section{Acknowledgments}

This work was supported within the CNR-CCLRC Agreement no. 01/9001 concerning collaboration in scientific research at the spallation neutron source ISIS. The financial support of the
Consiglio Nazionale delle Ricerche in this research is hereby acknowledged.

\section{References}

[1] G.C. Windsor, Pulsed Neutron Scattering, Taylor \& Francis Ltd., London, 1981.

[2] C. Andreani, D. Colognesi, J. Mayers, G.F. Reiter, R. Senesi, Advances in Physics 54 (2005) 377.

[3] G.K. Ivanov, Y.S. Sayasov, Soviet Physics Uspekhi 9 (1967) 670.

[4] P.C. Hohenberg, P.M. Platzman, Physical Review 152 (1966) 198.

[5] G.I. Watson, Journal of Physics: Condensed Matter 8 (1996) 5955.

[6] V.F. Sears, Physical Review B 30 (1984) 44.

[7] U. Bafile, M. Celli, M. Zoppi, J. Mayers, Physical Review B 58 (1998) 791

[8] U. Bafile, M. Zoppi, F. Barocchi, R. Magli, J. Mayers, Physical Review Letters 75 (1995) 1957.

[9] D.M. Ceperley, R.O. Simmons, R.C. Blasdell, Physical Review Letters 77 (1996) 115.

[10] R. Senesi, C. Andreani, D. Colognesi, A. Cunsolo, M. Nardone, Physical Review Letters 86 (2001) 4584.

[11] G. Reiter, C. Burnham, D. Homouz, P.M. Platzman, J. Mayers, T. Abdul-Redah, A.P. Moravsky, J.C. Li, C.-K. Loong, A.I. Kolesnikov, Physical Review Letters 97 (2006) 247801.

[12] M. Krzystyniak, M.A. Adams, A. Lovell, N.T. Skipper, S.M. Bennington, J. Mayers, F. Fernandez-Alonso, Faraday Discussions 151 (2011) 171

[13] J.V. Pearce, S.O. Diallo, H.R. Glyde, R.T. Azuah, T. Arnold, J.Z. Larese, Journal of Physics: Condensed Matter 16 (2004) 4391

[14] J.A. Morrone, L. Lin, R. Car, Journal of Chemical Physics 130 (2009) 204511.

[15] A. Pietropaolo, R. Senesi, C. Andreani, J. Mayers, Brazilian Journal of Physics 39 (2009) 318.

[16] R. Moreh, D. Nemirovsky, Journal of Chemical Physics 133 (2010) 084506.

[17] A.K. Soper, Physical Review Letters 103 (2009) 069801.

[18] D. Flammini, M.A. Ricci, F. Bruni, Journal of Chemical Physics 130 (2009) 236101.

[19] A. Pietropaolo, R. Senesi, C. Andreani, A. Botti, M.A. Ricci, F. Bruni, Physical Review Letters 103 (2009) 069802.

[20] A. Pietropaolo, R. Senesi, C. Andreani, A. Botti, M.A. Ricci, F. Bruni, Physical Review Letters 100 (2008) 127802.

[21] C. Pantalei, A. Pietropaolo, R. Senesi, S. Imberti, C. Andreani, J. Mayers, C. Burnham, G. Reiter, Physical Review Letters 100 (2008) 177801.

[22] A. Pietropaolo, C. Andreani, A. Filabozzi, R. Senesi, G. Gorini, E. Perelli-Cippo, M. Tardocchi, N.J. Rhodes, E.M. Schooneveld, Journal of Instrumentation 1 (2006) P04001.

[23] G.F. Reiter, J.C. Li, J. Mayers, T. Abdul-Redah, P. Platzman, Brazilian Journal of Physics 34 (2004) 142.

[24] C. Andreani, D. Colognesi, E. Degiorgi, M.A. Ricci, Journal of Chemical Physics 115 (2001) 11243.

[25] C. Andreani, E. Degiorgi, R. Senesi, F. Cilloco, D. Colognesi, J. Mayers, M. Nardone, E. Pace, Journal of Chemical Physics 114 (2001) 387.

[26] V. Garbuio, C. Andreani, S. Imberti, A. Pietropaolo, G.F. Reiter, R. Senesi, M.A. Ricci, Journal of Chemical Physics 127 (2007) 4501.

[27] R. Senesi, A. Pietropaolo, A. Bocedi, S.E. Pagnotta, F. Bruni, Physical Review Letters 98 (2007) 138102.

[28] G.F. Reiter, R. Senesi, J. Mayers, Physical Review Letters 105 (2010) 148101.

[29] C. Pantalei, R. Senesi, C. Andreani, P. Sozzani, A. Comotti, S. Bracco, M. Beretta, P.E. Sokol, G. Reiter, Physical Chemistry Chemical Physics 13 (2011) 6022.

[30] R. Senesi, C. Andreani, Z. Bowden, D. Colognesi, E. Degiorgi, A.L. Fielding, J. Mayers, M. Nardone, J. Norris, M. Praitano, N.J. Rhodes, W.G. Stirling, J. Tomkinson, C. Uden, Physica B: Condensed Matter 276 (2000) 200.

[31] J. Mayers, M.A. Adams, Nuclear Instruments and Methods in Physics Research A 625 (2011) 47.

[32] C. Andreani, D. Colognesi, E. Degiorgi, A. Filabozzi, M. Nardone, E. Pace, A. Pietropaolo, R. Senesi, Nuclear Instruments and Methods in Physics Research A 497 (2003) 535.

[33] S. Imberti, C. Andreani, V. Garbuio, G. Gorini, A. Pietropaolo, R. Senesi, M. Tardocchi, Nuclear Instruments and Methods in Physics Research A 522 (2005) 463.

[34] A. Pietropaolo, C. Andreani, A. Filabozzi, E. Pace, R. Senesi, Nuclear Instruments and Methods in Physics Research A 570 (2007) 498.

[35] E.M. Schooneveld, J. Mayers, N.J. Rhodes, A. Pietropaolo, C. Andreani, R. Senesi, G. Gorini, E. Perelli-Cippo, M. Tardocchi, Review of Scientific Instruments 77 (2006) 5103.

[36] J. Mayers, Measurement Science and Technology 22 (2011) 015903.

[37] C. Andreani, A. Pietropaolo, R. Senesi, G. Gorini, E. Perelli-Cippo, M. Tardocchi, N. Rhodes, E.M. Schooneveld, Applied Physics Letters 85 (2004) 5454.

[38] M. Tardocchi, G. Gorini, A. Pietropaolo, C. Andreani, R. Senesi, N. Rhodes, E.M. Schooneveld, Review of Scientific Instruments 75 (2004) 4880.

[39] C. Andreani, A. Pietropaolo, R. Senesi, G. Gorini, M. Tardocchi, A. Bracco, N. Rhodes, E. Schooneveld, Nuclear Instruments and Methods in Physics Research A 481 (2002) 509.

[40] M. Tardocchi, A. Pietropaolo, C. Andreani, A. Bracco, A. D’Angelo, G. Gorini, S. Imberti, R. Senesi, N.J. Rhodes, E.M. Schooneveld, Nuclear Instruments and Methods in Physics Research A 526 (2004) 477.

[41] C. Andreani, A. D’Angelo, G. Gorini, S. Imberti, A. Pietropaolo, N.J. Rhodes, E.M. Schooneveld, R. Senesi, M. Tardocchi, Applied Physics A: Materials Science \& Processing 78 (2004) 903. 
[42] J. Mayers, A.L. Fielding, R. Senesi, Nuclear Instruments and Methods in Physics Research A 481 (2002) 454.

[43] R.C. Blasdell, R.O. Simmons, Nuclear Instruments and Methods in Physics Research A 405 (1998) 71

[44] S.O. Diallo, R.T. Azuah, O. Kirichek, J.W. Taylor, H.R. Glyde, Physical Review B 80 (2009) 060504

[45] S.O. Diallo, J.V. Pearce, R.T. Azuah, O. Kirichek, J.W. Taylor, H.R. Glyde, Physical Review Letters 98 (2007) 205301.

[46] S.O. Diallo, J.V. Pearce, R.T. Azuah, F. Albergamo, H.R. Glyde, Physical Review B 74 (2006) 144503.

[47] D.G. Narehood, M.K. Kostov, P.C. Eklund, M.W. Cole, P.E. Sokol, Physica Review B 65 (2002) 233401.

[48] K.W. Herwig, P.E. Sokol, T.R. Sosnick, W.M. Snow, R.C. Blasdell, Physical Review B 41 (1990) 103.

[49] D. Homouz, G. Reiter, J. Eckert, J. Mayers, R. Blinc, Physical Review Letters 98 (2007) 115502.

[50] S.O. Diallo, J.V. Pearce, R.T. Azuah, H.R. Glyde, Physical Review Letters 93 (2004) 075301.
[51] R. Senesi, D. Colognesi, A. Pietropaolo, T. Abdul-Redah, Physical Review B 72 (2005) 054119.

[52] J. Mayers, T. Abdul-Redah, Journal of Physics Condensed Matter 16 (2004) 4811.

[53] D. Flammini, et al., Spherical momentum distribution of the protons in hexagonal ice from modeling of inelastic neutron scattering data, Journal of Chemical Physics, submitted for publication.

[54] L. Lin, J.A. Morrone, R. Car, M. Parrinello, Physical Review Letters 105 (2010) 110602.

[55] S.W. Lovesey, The Theory of Neutron Scattering from Condensed Matter, Oxford University Press, Oxford, 1986

[56] H.R. Glyde, Excitations in Liquid and Solid Helium, Clarendon, Oxford, 1994.

[57] R. Senesi, A. Pietropaolo, C. Andreani, Nuclear Instruments and Methods in Physics Research A 594 (2008) 244.

[58] E.T. Jaynes, G.T. Bretthorst, Probability Theory: Principles and Elementary Applications, vol. 1, Cambridge University Press, Cambridge, 2003. 